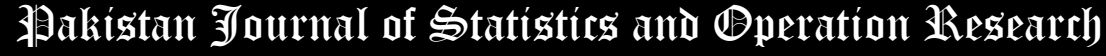

\section{The Discrete Type-II Half-Logistic Exponential Distribution with Applications to COVID-19 Data}

\author{
Muhammad Ahsan-ul-Haq ${ }^{1 *}$, Ayesha Babar ${ }^{2}$, Sharqa Hashmi ${ }^{3}$, Abdulaziz S. \\ Alghamdi $^{4}$, Ahmed Z. Afify ${ }^{5}$
}

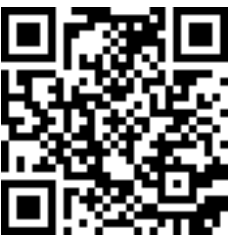

* Corresponding Author

1. College of Statistical \& Actuarial Sciences, University of the Punjab, Pakistan; ahsanshani36@gmail.com

2. School of Statistics, Minhaj University Lahore, Pakistan; ayeshababar19@gmail.com

3. Department of Statistics, Lahore College for Women University, Lahore Pakistan; sharqa.hashmi@gmail.com

4. Department of Mathematics, College of Science \& Arts, King Abdulaziz University, P.O. Box 344, Rabigh

21911, Saudi Arabia; ashalghamedi@kau.edu.sa

5. Department of Statistics, Mathematics and Insurance, Benha University, Benha 13511, Egypt; ahmed.afify@fcom.bu.edu.eg

\begin{abstract}
We propose a new two-parameter discrete model, called discrete Type-II half-logistics exponential (DTIIHLE) distribution using the survival discretization approach. The DTIIHLE distribution can be utilized to model COVID19 data. The model parameters are estimated using the maximum likelihood method. A simulation study is conducted to evaluate the performance of the maximum likelihood estimators. The usefulness of the proposed distribution is evaluated using two real-life COVID-19 data sets. The DTIIHLE distribution provides a superior fit to COVID-19 data as compared with competitive discrete models including the discrete-Pareto, discrete Burr-XII, discrete log-logistic, discrete-Lindley, discrete-Rayleigh, discrete inverse-Rayleigh, and natural discrete-Lindley.
\end{abstract}

Key Words: COVID-19 data; Discretization; maximum likelihood estimation; type II half logistics exponential; simulations

Mathematical Subject Classification: 62E15, 62F10, 62Pxx, 62Q05

\section{Introduction}

In late 2019, a novel coronavirus disease (COVID-19) was first reported in China and has been announced as an epidemic by the World Health Organization (WHO) (Lee et al., 2020). The epidemic has mostly been controlled in China since March 2020 but continues to inflict public health and socioeconomic situations in all other countries of the world. One of the major reasons for controlling the disease is China's strategy of effective use of its health care system and publicity of awareness programs among people which played a vital role in the control of the COVID-19 pandemic. However, the major source of its rapid spread is human-to-human contact.

It is well recognized that the life duration in the real world is related to continuous non-negative lifetime distributions. However, it is sometimes uneasy to obtain the samples from a continuous distribution. The observed data obtained are discrete because they are usually measured at only a finite number of decimal places and can not assume all points within an interval. When measures are taken on a continuous (ratio or interval) scale, the discrete distributions are more appropriate for such observations. Therefore, it is rational to assume that these observations are from a discretized distribution which is constructed from a continuous distribution same (Chakraborty, 2015). 
During the last few decades, many continuous lifetime distributions have been proposed and studied. However, research work on discrete distributions is not widely addressed comparatively to continuous distributions. The discretization of continuous lifetime models has been applied to derive discrete lifetime distributions. The discretization of continuous distributions keeps similar functional form of the survival function (SF), as well as resulting in many reliability properties which remain the same (Nakagawa and Osaki, 1975).

Recently, the methods of generating discrete analogues of continuous distributions have been considered by several authors. For example, the infinite series discretization method (Good, 1953; Kulasekera and Tonkyn, 1992; Kemp,1997; Sato et al., 1999), survival discretization approach has an interesting feature of keeping the original functional form of SF (Nakagawa and Osaki, 1975), hazard function discretization approach (Stein, 1984), compound two-phase method (Chakraborty, 2015), reversed hazard function discretization method (Ghosh et al., 2013).

Some notable recent proposed discrete distributions include discrete Weibull (Nakagawa and Osaki, 1975), discrete skew-laplace (Kozubowski and Inusah, 2006), discrete-laplace (Inusah and Kozubowski, 2006), discreteMaxwell (Krishna and Pundir, 2007), discrete-Burr (Krishna and Pundir, 2009), discrete inverse-Weibull (Jazi, 2010), discrete-Lindley (Ojeda, 2011), discrete-gamma (Chakraborty and Chakravarty, 2012), discrete Burr-III (Al-Huniti, 2012), discrete inverse-Rayleigh (Hussain and Ahmad, 2014), discrete generalized-Rayleigh (Alamatsaz et al., 2016), natural discrete-Lindley (Al-Babtain et al., 2020), transmuted record type geometric (Almazah et al., 2021), discrete inverted Topp-Leone (Eldeeb et al., 2021), and uniform Poisson-Ailamujia (Aljohani et al., 2021), among many others.

The main objective of this article is to provide a new flexible two-parameter discrete model, called the discrete type-II half-logistics exponential (DTIIHLE) distribution using the survival discretization approach. The DTIIHLE distribution can be utilized to model over-dispersed count data sets. Its hazard rate function (HRF) can be decreasing or unimodal. We derive some of its properties in explicit forms such as the quantile function $(\mathrm{QF})$, moments and probability generating function (PGF). The two parameters are estimated via the maximum likelihood (ML) and a simulation study is conducted to explore the performance of the ML estimators. The importance of the newly DTIIHLE distribution is illustrated by analyzing two real-life COVID-19 data sets which represent the number of COVID-19 deaths in Pakistan and Saudi Arabia.

The rest of the article is structured as follows. In Section 2, the DTIIHLE distribution is defined with some plots of its probability mass function (PMF) and HRF. Some properties of the DTIIHLE distribution are provided in Section 3. The ML approach is adopted to estimate the DTIIHLE parameters in Section 4. Simulation results are conducted to explore the behavior of the introduced estimators in Section 5. To validate the use of DTIIHLE distribution in fitting real-life count data, two sets of data from medicine field are fitted in Section 6. Finally, some conclusions are presented in Section 7.

\section{The DTIIHLE Distribution}

The SF of type II half-logistic exponential (TIIHLE) distribution (Elgarhy et al., 2019) takes the form

$$
S(x)=\frac{1-\left(1-e^{-\delta x}\right)^{\lambda}}{1+\left(1-e^{-\delta x}\right)^{\lambda}}, x>0, \delta, \lambda>0,
$$

where $\delta$ is scale and $\lambda$ is the shape parameters.

The probability density function of the TIIHLE distribution is

$$
f(x)=\frac{2 \delta \lambda e^{-\delta x}\left(1-e^{-\delta x}\right)^{\lambda-1}}{\left[1+\left(1-e^{-\delta x}\right)^{\lambda}\right]^{2}}
$$


A discrete analog of any continuous random variable can be obtained using different discretization approaches. A review on such discretization techniques can be explored in Chakraborty (2015). The most common discretization method is the one preserving the functional form of the SF. Let $X$ be a continuous random variable (RV) with $\mathrm{SF} S(x)$. The corresponding PMF of a discrete RV reduces to

$$
P(X=x)=S(x)-S(x+1), \quad x=0,1,2,3, \ldots
$$

To this end, we apply this discretization method of the continuous TIIHLE distribution to generate the corresponding DTIIHLE model which is defined by the PMF

$$
P(x)=\frac{2\left[\left(1-\theta^{x+1}\right)^{\lambda}-\left(1-\theta^{x}\right)^{\lambda}\right]}{\left[1+\left(1-\theta^{x}\right)^{\lambda}\right]\left[1+\left(1-\theta^{x+1}\right)^{\lambda}\right]}, \quad x=0,1,2,3, \ldots, \lambda>0, \quad 0<\theta<1,
$$

where $e^{-\delta}=\theta$ and $0<\theta<1$.

The corresponding cumulative distribution function (CDF), $F(x)=P(X \leq x)$, of DTIIHLE distribution takes the form

$$
F(x)=1-P(X>x)=\frac{2\left(1-\theta^{x+1}\right)^{\lambda}}{1+\left(1-\theta^{x+1}\right)^{\lambda}} .
$$

From Equation (5), one can easily derive the QF as follows

$$
x_{p}=\frac{\log \left(1-\left(\frac{p}{2-p}\right)^{\frac{1}{\lambda}}\right)}{\log \theta}, 0<p<1
$$

The SF and HRF of the DTIIHLE model are given as

$$
S(x)=\frac{1-\left(1-\theta^{x+1}\right)^{\lambda}}{1+\left(1-\theta^{x+1}\right)^{\lambda}}
$$

and

$$
h(x)=\frac{2\left[\left(1-\theta^{x+1}\right)^{\lambda}-\left(1-\theta^{x}\right)^{\lambda}\right]}{\left[1+\left(1-\theta^{x}\right)^{\lambda}\right]\left[1-\left(1-\theta^{x+1}\right)^{\lambda}\right]} .
$$

The reverse HRF and the second rate of failure of the DTIIHLE model are defined by

$$
r^{*}(x)=\frac{P(x)}{F(x)}=\frac{\left(1-\theta^{x+1}\right)^{\lambda}-\left(1-\theta^{x}\right)^{\lambda}}{\left(1-\theta^{x+1}\right)^{\lambda}\left[1+\left(1-\theta^{x}\right)^{\lambda}\right]}
$$

and

$$
r^{* *}(\mathrm{x})=\log \left(\frac{\left\{1-\left[1-\theta^{x+1}\right]^{\lambda}\right\}\left\{1+\left[1-\theta^{x+2}\right]^{\lambda}\right\}}{\left\{1+\left[1-\theta^{x+1}\right]^{\lambda}\right\}\left\{1-\left[1-\theta^{x+2}\right]^{\lambda}\right\}}\right) .
$$

The recurrence relation which can be used to generate probabilities from the DTIIHLE distribution has the form

$$
P(x+1)=\frac{\left[1+\left(1-\theta^{x}\right)^{\lambda}\right]\left[\left(1-\theta^{x+2}\right)^{\lambda}-\left(1-\theta^{x+1}\right)^{\lambda}\right]}{\left[1+\left(1-\theta^{x+2}\right)^{\lambda}\right]\left[\left(1-\theta^{x+1}\right)^{\lambda}-\left(1-\theta^{x}\right)^{\lambda}\right]} P(x)
$$

The PMF plots for different values of its parameters are presented in Figure 1. The HRF plots are displayed in Figure 2. The plots reveal that its PMF can be unimodal, as well as its HRF can be decreasing or unimodal. 

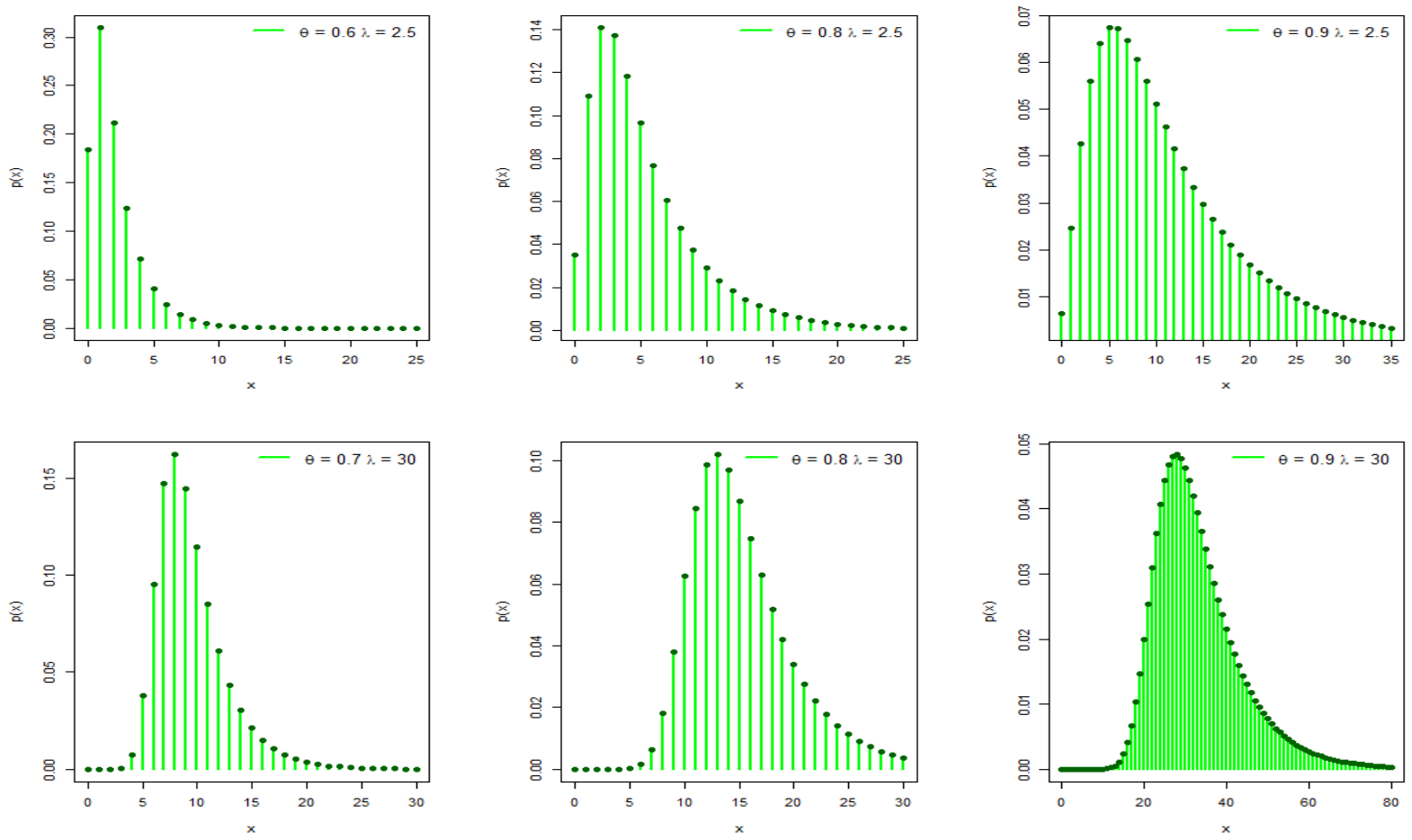

Figure 1: Some plots for the PMF of the DTIIHLE distribution for selected parameters.
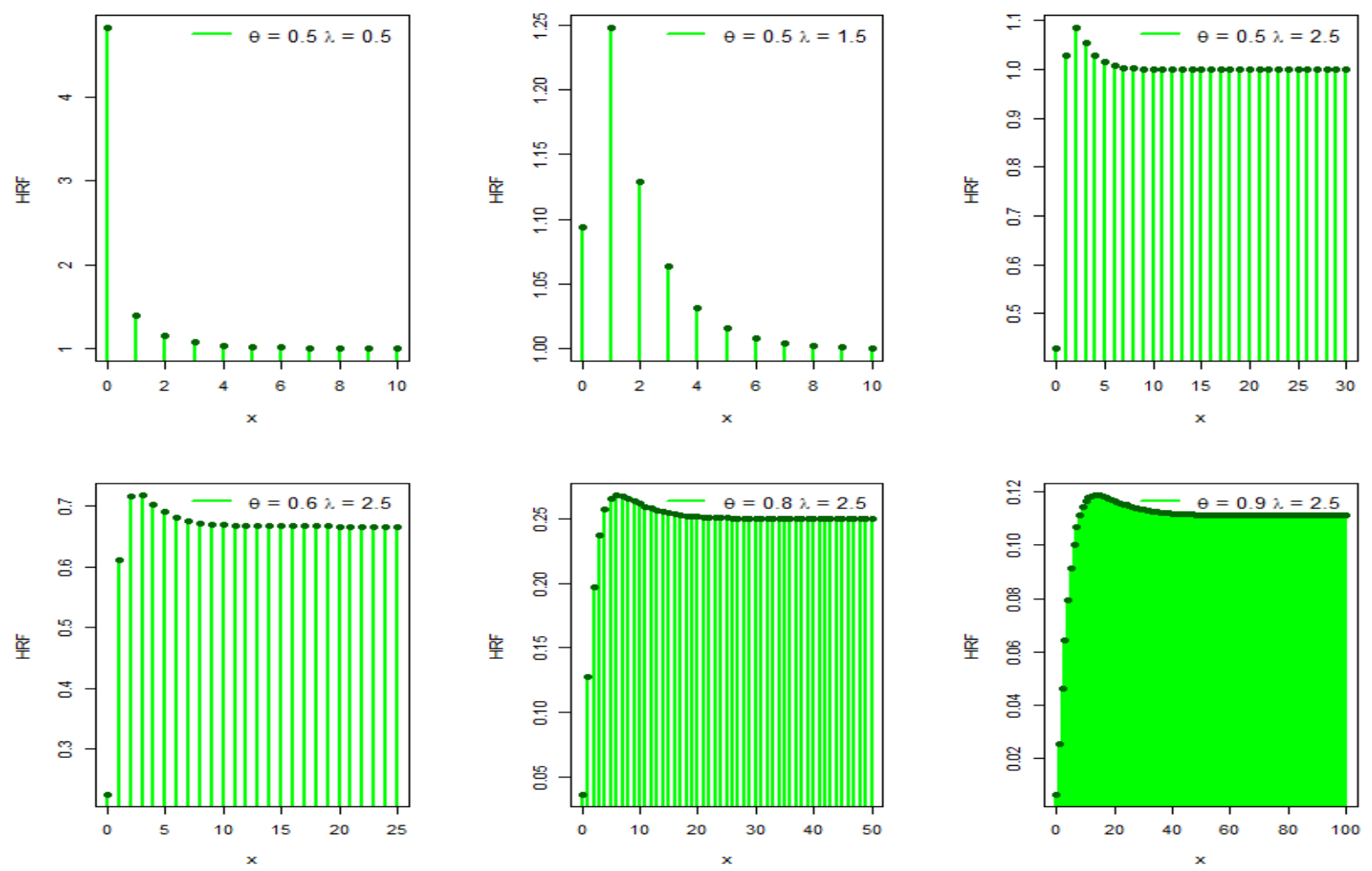

Figure 2: The HRF plots of DTIIHLE distribution for selected parameters.

\section{The PGF and moments}


The PGF of the DTIIHLE distribution follows as

$$
\begin{gathered}
\mathrm{G}_{x}(Z)=\sum_{x=0}^{\infty} Z^{x} \mathrm{P}(x)=\sum_{x=0}^{\infty} Z^{x}[S(x)-S(x+1)], \\
\mathrm{G}_{x}(Z)=1+(Z-1) \sum_{x=1}^{\infty} Z^{x-1} S(x), \\
\mathrm{G}_{x}(Z)=1+(Z-1) \sum_{x=1}^{\infty} Z^{x-1} \frac{1-\left(1-\theta^{x+1}\right)^{\lambda}}{1+\left(1-\theta^{x+1}\right)^{\lambda}} .
\end{gathered}
$$

Differentiating $\mathrm{G}_{x}(Z)$ with respect to $Z$ and setting $Z=1$, we obtain the mean of DTIIHLE distribution as

$$
\begin{gathered}
\mathrm{G}^{\prime} \mathrm{x}(\mathrm{Z})=(1) \sum_{x=1}^{\infty} Z^{x-1} \frac{1-\left(1-\theta^{x+1}\right)^{\lambda}}{1+\left(1-\theta^{x+1}\right)^{\lambda}}+(Z-1) \sum_{x=1}^{\infty}(x-1) Z^{x-2} \frac{1-\left(1-\theta^{x+1}\right)^{\lambda}}{1+\left(1-\theta^{x+1}\right)^{\lambda}}, \\
\mathrm{G}^{\prime} \mathrm{x}(1)=\sum_{x=1}^{\infty} \frac{1-\left(1-\theta^{x+1}\right)^{\lambda}}{1+\left(1-\theta^{x+1}\right)^{\lambda}}
\end{gathered}
$$

Again differentiate $G_{X}^{\prime}(Z)$ with respect to (wrt) $Z$ and setting $Z=1$, we obtain

$$
\mathrm{G}^{\prime \prime} \mathrm{x}(1)=2 \sum_{x=1}^{\infty}(x-1) \frac{1-\left(1-\theta^{x+1}\right)^{\lambda}}{1+\left(1-\theta^{x+1}\right)^{\lambda}} .
$$

On differentiating G"x (Z) wrt $Z$ and setting $Z=1$, we have

$$
G^{\prime \prime \prime} x(1)=3 \sum_{x=1}^{\infty}(x-2)(x-1) \frac{1-\left(1-\theta^{x+1}\right)^{\lambda}}{1+\left(1-\theta^{x+1}\right)^{\lambda}} .
$$

On differentiating $\mathrm{G}^{\prime \prime \prime}{ }_{\mathrm{x}}(\mathrm{Z})$ wrt $\mathrm{Z}$ and putting $\mathrm{Z}=1$, we get

$$
\mathrm{G}^{\prime \prime \prime} \mathrm{x}(1)=4 \sum_{x=1}^{\infty}(x-3)(x-2)(x-1) \frac{1-\left(1-\theta^{x+1}\right)^{\lambda}}{1+\left(1-\theta^{x+1}\right)^{\lambda}} .
$$

Moments about the origin can be calculated using the factorial moments as

$$
\begin{gathered}
\mu=G^{\prime} x(1)=\sum_{x=1}^{\infty} \frac{1-\left(1-\theta^{x+1}\right)^{\lambda}}{1+\left(1-\theta^{x+1}\right)^{\lambda}} \\
\mu_{2}^{\prime}=\sum_{x=1}^{\infty}(2 x-1) \frac{1-\left(1-\theta^{x+1}\right)^{\lambda}}{1+\left(1-\theta^{x+1}\right)^{\lambda}} \\
\mu_{3}^{\prime}=\sum_{x=1}^{\infty}\left(3 x^{2}-3 x+1\right) \frac{1-\left(1-\theta^{x+1}\right)^{\lambda}}{1+\left(1-\theta^{x+1}\right)^{\lambda}} \\
\mu_{4}^{\prime}=\sum_{x=1}^{\infty}\left(4 x^{3}-6 x^{2}+4 x-1\right) \frac{1-\left(1-\theta^{x+1}\right)^{\lambda}}{1+\left(1-\theta^{x+1}\right)^{\lambda}} .
\end{gathered}
$$

Now variance is 


$$
\sigma^{2}=\mu_{2}^{\prime}-\left(\mu_{1}^{\prime}\right)^{2}=\sum_{x=1}^{\infty}(2 x-1) \frac{1-\left(1-\theta^{x+1}\right)^{\lambda}}{1+\left(1-\theta^{x+1}\right)^{\lambda}}-\left(\sum_{x=1}^{\infty} \frac{1-\left(1-\theta^{x+1}\right)^{\lambda}}{1+\left(1-\theta^{x+1}\right)^{\lambda}}\right)^{2} .
$$

The coefficient of skewness and kurtosis can be calculated by the formulae

$$
S K=\frac{\mu_{3}}{\left(\sigma^{2}\right)^{\frac{3}{2}}}=\frac{\mu_{3}^{\prime}-3 \mu_{2}^{\prime} \mu+2 \mu^{3}}{\left(\sigma^{2}\right)^{\frac{3}{2}}}
$$

and

$$
C K=\frac{\mu_{4}^{\prime}-4 \mu_{3}^{\prime} \mu+6 \mu_{2}^{\prime} \mu^{2}-3 \mu^{4}}{\left(\sigma^{2}\right)^{2}}
$$

The dispersion index (DI) is defined by $D I=\sigma^{2} / \mu$.

Table 1 shows descriptive measures of the DTIIHLE distribution for different parameter values. One can note that the skewness decreases as the value of the shape parameter increases. If the value of the DI is greater than 1, then the proposed distribution is applicable for over-dispersed data.

Table 1: Some descriptive measures of DTIIHLE distribution for selected parameter values.

\begin{tabular}{cccccc}
\hline$(\lambda, \theta)$ & Mean & Variance & Skewness & Kurtosis & DI \\
\hline$(0.5,0.2)$ & 0.06844 & 0.09545 & 5.66623 & 44.59604 & 1.39465 \\
$(0.5,0.5)$ & 0.30866 & 0.74495 & 4.22006 & 27.90377 & 2.41346 \\
$(0.5,0.7)$ & 0.77976 & 3.18343 & 3.81945 & 23.94728 & 4.08255 \\
$(0.5,0.9)$ & 3.28509 & 39.4304 & 3.57159 & 21.77556 & 12.0028 \\
$(1.0,0.2)$ & 0.13654 & 0.18128 & 3.84640 & 22.13221 & 1.32770 \\
$(1.0,0.5)$ & 0.60670 & 1.29990 & 2.87381 & 15.10421 & 2.14260 \\
$(1.0,0.7)$ & 1.50133 & 5.23722 & 2.67496 & 14.16982 & 3.48838 \\
$(1.0,0.9)$ & 6.09633 & 61.4726 & 2.60192 & 14.27673 & 10.0835 \\
$(1.5,0.2)$ & 0.20394 & 0.25742 & 3.01399 & 14.79993 & 1.26219 \\
$(1.5,0.5)$ & 0.88562 & 1.68872 & 2.30202 & 11.57471 & 1.90683 \\
$(1.5,0.7)$ & 2.13358 & 6.48712 & 2.23105 & 12.14343 & 3.04048 \\
$(1.5,0.9)$ & 8.37513 & 74.2518 & 2.23195 & 13.36564 & 8.86576 \\
\hline
\end{tabular}

\section{Parameter Estimation}

Let $x_{1}, x_{2}, x_{3}, \ldots, x_{n}$ be a random sample of size $n$ from the DTIIHLE model. Then, the log-likelihood function is given by

$L=n \ln 2+\sum_{i=1}^{n} \ln \left(\left(1-\theta^{x_{i}+1}\right)^{\lambda}-\left(1-\theta^{x_{i}}\right)^{\lambda}\right)-\sum_{i=1}^{n} \ln \left[1+\left(1-\theta^{x_{i}}\right)^{\lambda}\right]-\sum_{i=1}^{n} \ln \left[1+\left(1-\theta^{x_{i}+1}\right)^{\lambda}\right]$.

The first derivatives wrt $\theta$ and $\lambda$ are

$$
\begin{gathered}
\frac{\partial L}{\partial \theta}=-\sum_{i=1}^{n} \frac{x_{i} \theta^{x_{i}-1} \lambda\left(1-\theta^{x_{i}+1}\right)^{\lambda}-\lambda\left(x_{i}+1\right) \theta^{x_{i}}\left(1-\theta^{x_{i}}\right)^{\lambda}}{\left(1-\theta^{x_{i}+1}\right)^{\lambda}-\left(1-\theta^{x_{i}}\right)^{\lambda}}+\sum_{i=1}^{n} \frac{\lambda x_{i} \theta^{x_{i}-1}\left(1-\theta^{x_{i}}\right)^{\lambda-1}}{1+\left(1-\theta^{x_{i}}\right)^{\lambda}} \\
+\sum_{i=1}^{n} \frac{\lambda\left(x_{i}+1\right) \theta^{x_{i}}\left(1-\theta^{x_{i}+1}\right)^{\lambda-1}}{1+\left(1-\theta^{x_{i}+1}\right)^{\lambda}}
\end{gathered}
$$


and

$$
\begin{gathered}
\frac{\partial L}{\partial \lambda}=\sum_{i=1}^{n} \frac{\left(1-\theta^{x_{i}+1}\right)^{\lambda} \ln \left(1-\theta^{x_{i}+1}\right)-\left(1-\theta^{x_{i}}\right)^{\lambda} \ln \left(1-\theta^{x_{i}}\right)}{\left(1-\theta^{x_{i}+1}\right)^{\lambda}-\left(1-\theta^{x_{i}}\right)^{\lambda}}-\sum_{i=1}^{n} \frac{\left(1-\theta^{x_{i}}\right)^{\lambda} \ln \left(1-\theta^{x_{i}}\right)}{1+\left(1-\theta^{x_{i}}\right)^{\lambda}} \\
-\sum_{i=1}^{n} \frac{\left(1-\theta^{x_{i}+1}\right)^{\lambda} \ln \left(1-\theta^{x_{i}+1}\right)}{1+\left(1-\theta^{x_{i}+1}\right)^{\lambda}} .
\end{gathered}
$$

The ML estimates (MLEs) of $\theta$ and $\lambda$ can be obtained using numerical methods.

\section{Simulation Study}

A comprehensive simulation study has been conducted by generating 10,000 samples of various sample sizes from the DTIIHLE distribution. Particularly, we generate the samples using the following combination of parameters $(\theta, \lambda)$ i.e., $(0.50,0.50),(0.50,1.50),(0.50,2.50),(0.50,3.0),(0.80,0.50),(0.80,1.50),(0.50,2.50),(0.80,3.0)$.

The average estimates (MLEs), mean square errors (MSEs), and convergence probabilities are listed in Table 2. The MLEs are quite stable and very close to the true values of the parameters. The MLEs are consistent as shown from Table 2.

\section{Modeling COVID-19 Data}

In this section, we illustrate the importance of the newly DTIIHLE distribution by utilizing two real-life data sets. We compared the fits of the DTIIHLE model with the following competitive distributions: discrete Burr XII (DBXII) and discrete Pareto (DPr) (Krishna and Pundir, 2009), discrete log-logistic (DLoL) (Para and Jan, 2016), discrete Rayleigh (DR) (Roy, 2004), discrete-Lindley (DL) (Bakouch et al., 2014), discrete inverse-Rayleigh (DIR) (Hussain and Ahmad, 2014), discrete Burr-Hutke (DBH) (El-Morshedy et al., 2020), discrete Bilal (DBi) (Altun et al., 2020), and natural discrete Lindley (NDL) (Al-Babtain et al., 2020).

The first data refer to number of coronavirus deaths in Pakistan from 19 March 2020 to 30 June 2020. The data set is reported at: https://www.worldometers.info/ . The data are: 1, 0, 2, 1, 1, 1, 1, 2, 1, 2, 7, 5, 1, $7,6,1,6,6,4,4,4,1,20,5,2,3,15,17,7,8,25,8,25,11,25,16,16,12,11,20,31,42,32$, $23,17,19,38,50,21,14,37,23,47,31,24,9,64,0,39,30,36,46,32,50,34,32,34,30,28,35$, 57, 78, 88, 60, 78, 67, 82, 68, 97, 67, 65, 105, 83, 101, 107, 0, 88, 178, 110, 136, 118, 136, 153, 119, 89, 105, $60,148,59,73,83,49,137,91$. The second data refer to number of deaths due to coronavirus in Saudi Arabia from 19 March 2020 to 30 June 2020. The data are: $1,1,1,0,1,4,0,2,6,5,4,4,5,4,3,3,3,5,7,6,8,6,4,4,5,5,6,6$, $5,7,6,9,3,5,8,5,5,7,7,8,7,9,9,10,10,10,7,9,9,9,10,9,10,10,8,9,10,12,13,15,11,9,12,14,16,17,22$, $23,22,24,30,32,31,34,36,34,37,36,38,36,39,40,39,41,39,48,45,46,37,40,39,41,41,46,37,40,48,50$, 49.

The MLEs, standard errors (SE) and 95\% confidence intervals (C.I.) for the estimates are listed in Tables 3 and 5 for the two data sets, respectively. Some goodness-of-fit measures including log-likelihood $(\ell)$, AIC, BIC and KS statistic are presented in Tables 4 and 6 for the respective two data sets. 
Table 2: Simulation results of selected values of parameters of the DTIIHLE model

\begin{tabular}{|c|c|c|c|c|c|c|c|}
\hline \multirow{2}{*}{ Parameters } & \multirow{2}{*}{$\mathbf{n}$} & \multicolumn{2}{|c|}{ MLES } & \multicolumn{2}{|c|}{ MSEs } & \multicolumn{2}{|c|}{$95 \% \mathrm{CP}$} \\
\hline & & $\widehat{\boldsymbol{\theta}}$ & $\hat{\lambda}$ & $\widehat{\boldsymbol{\theta}}$ & $\hat{\lambda}$ & $\widehat{\boldsymbol{\theta}}$ & $\hat{\lambda}$ \\
\hline \multirow{5}{*}{$\theta=0.5, \lambda=0.5$} & 20 & 0.4105 & 27.391 & 0.0651 & 26.891 & 0.8125 & 0.7880 \\
\hline & 50 & 0.4617 & 1.7259 & 0.0254 & 1.2259 & 0.9082 & 0.9330 \\
\hline & 100 & 0.4773 & 0.7310 & 0.0141 & 0.2310 & 0.9187 & 0.9431 \\
\hline & 200 & 0.4881 & 0.5408 & 0.0067 & 0.0408 & 0.9351 & 0.9497 \\
\hline & 300 & 0.4919 & 0.5251 & 0.0044 & 0.0251 & 0.9393 & 0.9536 \\
\hline \multirow{5}{*}{$\theta=0.5, \lambda=1.5$} & 20 & 0.4602 & 3.9690 & 0.0232 & 2.4690 & 0.9003 & 0.9406 \\
\hline & 50 & 0.4834 & 1.7708 & 0.0091 & 0.2708 & 0.9296 & 0.9504 \\
\hline & 100 & 0.4913 & 1.5882 & 0.0044 & 0.0882 & 0.9418 & 0.9526 \\
\hline & 200 & 0.4955 & 1.5433 & 0.0022 & 0.0433 & 0.9458 & 0.9477 \\
\hline & 300 & 0.4977 & 1.5251 & 0.0014 & 0.0251 & 0.9459 & 0.9513 \\
\hline \multirow{5}{*}{$\theta=0.5, \lambda=2.5$} & 20 & 0.4748 & 3.1482 & 0.0136 & 0.6482 & 0.9141 & 0.9462 \\
\hline & 50 & 0.4894 & 2.7216 & 0.0054 & 0.2216 & 0.9358 & 0.9531 \\
\hline & 100 & 0.4945 & 2.6044 & 0.0026 & 0.1044 & 0.9433 & 0.9546 \\
\hline & 200 & 0.4973 & 2.5500 & 0.0012 & 0.0500 & 0.9478 & 0.9494 \\
\hline & 300 & 0.4981 & 2.5334 & 0.0008 & 0.0333 & 0.9497 & 0.9508 \\
\hline \multirow{5}{*}{$\theta=0.5, \lambda=3.0$} & 20 & 0.4766 & 3.8646 & 0.0117 & 0.8646 & 0.9227 & 0.9495 \\
\hline & 50 & 0.4895 & 3.2622 & 0.0045 & 0.2622 & 0.9363 & 0.9580 \\
\hline & 100 & 0.4951 & 3.1189 & 0.0023 & 0.1189 & 0.9379 & 0.9503 \\
\hline & 200 & 0.4979 & 3.0570 & 0.0011 & 0.0570 & 0.9503 & 0.9527 \\
\hline & 300 & 0.4980 & 3.0427 & 0.0007 & 0.0427 & 0.9439 & 0.9505 \\
\hline \multirow{5}{*}{$\theta=0.8, \lambda=0.5$} & 20 & 0.7483 & 0.7667 & 0.0197 & 0.2667 & 0.9316 & 0.9520 \\
\hline & 50 & 0.7804 & 0.5426 & 0.0056 & 0.0426 & 0.9452 & 0.9546 \\
\hline & 100 & 0.7905 & 0.5189 & 0.0024 & 0.0189 & 0.9440 & 0.9528 \\
\hline & 200 & 0.7948 & 0.5103 & 0.0011 & 0.0103 & 0.9530 & 0.9530 \\
\hline & 300 & 0.7967 & 0.5076 & 0.0007 & 0.0076 & 0.9468 & 0.9520 \\
\hline \multirow{5}{*}{$\theta=0.8, \lambda=1.5$} & 20 & 0.7786 & 1.7279 & 0.0052 & 0.2279 & 0.9374 & 0.9562 \\
\hline & 50 & 0.7922 & 1.5826 & 0.0016 & 0.0826 & 0.9446 & 0.9542 \\
\hline & 100 & 0.7958 & 1.5415 & 0.0007 & 0.0415 & 0.9508 & 0.9506 \\
\hline & 200 & 0.7981 & 1.5179 & 0.0004 & 0.0179 & 0.9496 & 0.9496 \\
\hline & 300 & 0.7986 & 1.5150 & 0.0002 & 0.0150 & 0.9502 & 0.9552 \\
\hline \multirow{5}{*}{$\theta=0.8, \lambda=2.5$} & 20 & 0.7823 & 2.9864 & 0.0034 & 0.4864 & 0.9436 & 0.9576 \\
\hline & 50 & 0.7933 & 2.6488 & 0.0011 & 0.1488 & 0.9490 & 0.9600 \\
\hline & 100 & 0.7961 & 2.5810 & 0.0005 & 0.0810 & 0.9490 & 0.9526 \\
\hline & 200 & 0.7984 & 2.5409 & 0.0003 & 0.0409 & 0.9494 & 0.9540 \\
\hline & 300 & 0.7988 & 2.5312 & 0.0002 & 0.0312 & 0.9488 & 0.9510 \\
\hline \multirow{5}{*}{$\theta=0.8, \lambda=3.0$} & 20 & 0.7819 & 3.6572 & 0.0031 & 0.6572 & 0.9434 & 0.9622 \\
\hline & 50 & 0.7933 & 3.2203 & 0.0011 & 0.2203 & 0.9438 & 0.9572 \\
\hline & 100 & 0.7968 & 3.1042 & 0.0005 & 0.1042 & 0.9456 & 0.9506 \\
\hline & 200 & 0.7983 & 3.0493 & 0.0002 & 0.0493 & 0.9542 & 0.9546 \\
\hline & 300 & 0.7990 & 3.0328 & 0.0002 & 0.0328 & 0.9506 & 0.9488 \\
\hline
\end{tabular}

From Tables 4 and 6, it is observed that the DTIIHLE distribution outperforms all other fitted models in analyzing number of deaths in Pakistan and Saudi Arabia. It can provide the best fit to the analyzed data among all other competitive distributions. Figures 3 and 4 display the PP plots for all the competitive distributions for the two data and they support the findings in Tables 4 and 6. 
Table 3: The MLEs, SE of the competing discrete distributions for the number of deaths in Pakistan

\begin{tabular}{lcccccc}
\hline \multirow{2}{*}{ Model } & \multicolumn{2}{c}{$\theta$} & \multicolumn{2}{c}{ 95\% C.I. } & \multicolumn{2}{c}{ 95\% C.I. } \\
\cline { 2 - 7 } & MLE & SE & & MLE & SE & \\
\hline DTIIHLE & 0.9850 & 0.0024 & {$[0.9803-0.9897]$} & 0.9188 & 0.1002 & {$[0.7224-1.1151]$} \\
DBXII & 0.9206 & 0.0439 & {$[0.8345-1.0066]$} & 3.9835 & 2.2732 & {$[0.4719-8.4389]$} \\
DLoL & 23.452 & 3.4905 & {$[16.611-30.294]$} & 1.1582 & 0.0955 & {$[0.9710-1.3454]$} \\
DL & 0.9553 & 0.0030 & {$[0.9494-0.9612]$} & - & - & - \\
NDL & 0.0443 & 0.0030 & {$[0.0384-0.0502]$} & - & - & - \\
DPr & 0.3190 & 0.0313 & {$[0.2577-0.3803]$} & - & - & - \\
DR & 42.402 & 2.0796 & {$[38.326-46.478]$} & - & - & - \\
DIR & 14.286 & 1.4987 & {$[11.349-17.223]$} & - & - & - \\
DBH & 0.9997 & 0.0016 & {$[0.9966-1.0028]$} & - & - & - \\
DBi & 50.692 & 3.5993 & {$[43.637-57.747]$} & - & - & - \\
\hline
\end{tabular}

Table 4: The goodness-of-fit criteria of the competing discrete distributions for the number of deaths in Pakistan

\begin{tabular}{lcccc}
\hline Model & $\ell$ & AIC & BIC & KS \\
\hline DTIIHLE & -493.94 & 991.87 & 997.16 & 0.4300 \\
DBXII & -541.81 & 1087.63 & 1092.91 & 0.0000 \\
DLo & -503.75 & 1011.49 & 1016.78 & 0.3400 \\
DL & -523.08 & 1048.16 & 1050.80 & 0.0002 \\
NDL & -520.27 & 1042.54 & 1045.18 & 0.0000 \\
DPr & -549.09 & 1100.19 & 1102.83 & 0.0000 \\
DR & -570.36 & 1142.72 & 1145.36 & 0.0000 \\
DIR & -717.98 & 1437.96 & 1440.61 & 0.0000 \\
DBH & -652.19 & 1306.38 & 1309.03 & 0.0000 \\
DBi & -530.64 & 1063.28 & 1065.92 & 0.0001 \\
\hline
\end{tabular}

Table 5: The MLEs, SE of the competing discrete distributions for the number of deaths in Saudi Arabia

\begin{tabular}{lcccccc}
\hline \multirow{2}{*}{ Model } & \multicolumn{2}{c}{$\theta$} & \multicolumn{2}{c}{$\lambda$ 95\% C.I. } & \multicolumn{1}{c}{ 95\% C.I. } \\
\cline { 2 - 7 } & MLE & SE & & MLE & SE & \\
\hline DTIIHLE & 0.94695 & 0.00732 & {$[0.9326-0.9613]$} & 1.52020 & 0.19062 & {$[1.1466-1.8938]$} \\
DBXII & 0.89516 & 0.05171 & {$[0.7938-0.9965]$} & 3.70604 & 1.90433 & {$[0.0264-7.4385]$} \\
DLoL & 10.2389 & 1.20670 & {$[7.8738-12.604]$} & 1.67939 & 0.13983 & {$[1.4053-1.9535]$} \\
DL & 0.89782 & 0.00689 & {$[0.8843-0.9113]$} & - & - & - \\
NDL & 0.10015 & 0.00678 & {$[0.0869-0.1134]$} & - & - & - \\
DPr & 0.39185 & 0.03938 & {$[0.3147-0.4690]$} & - & - & - \\
DR & 16.5490 & 0.83194 & {$[14.918-18.179]$} & - & - & - \\
DIR & 20.0897 & 2.09370 & {$[15.986-24.193]$} & - & - & - \\
DBH & 0.99818 & 0.00444 & {$[0.9895-1.0069]$} & - & - & - \\
DBi & 21.0474 & 1.53030 & {$[18.048-24.047]$} & - & - & - \\
\hline
\end{tabular}


Table 6: The goodness-of-fit measures of the competing discrete distributions for the of deaths in Saudi Arabia

\begin{tabular}{lcccc}
\hline Model & $\ell$ & AIC & BIC & KS \\
\hline DTIIHLE & -381.06 & 766.11 & 771.30 & 0.0390 \\
DBXII & -432.58 & 869.15 & 874.34 & 0.0000 \\
DLoL & -384.86 & 773.72 & 778.91 & 0.0140 \\
DL & -386.57 & 775.15 & 777.74 & 0.0002 \\
NDL & -385.81 & 773.62 & 776.22 & 0.0000 \\
DPr & -444.56 & 891.13 & 893.72 & 0.0000 \\
DR & -414.96 & 831.93 & 834.52 & 0.0000 \\
DIR & -467.63 & 937.26 & 939.85 & 0.0000 \\
DBH & -505.30 & 1012.6 & 1015.2 & 0.0000 \\
DBi & -388.88 & 779.77 & 782.36 & 0.0001 \\
\hline & DBXII & DLoL & DL & NDL
\end{tabular}
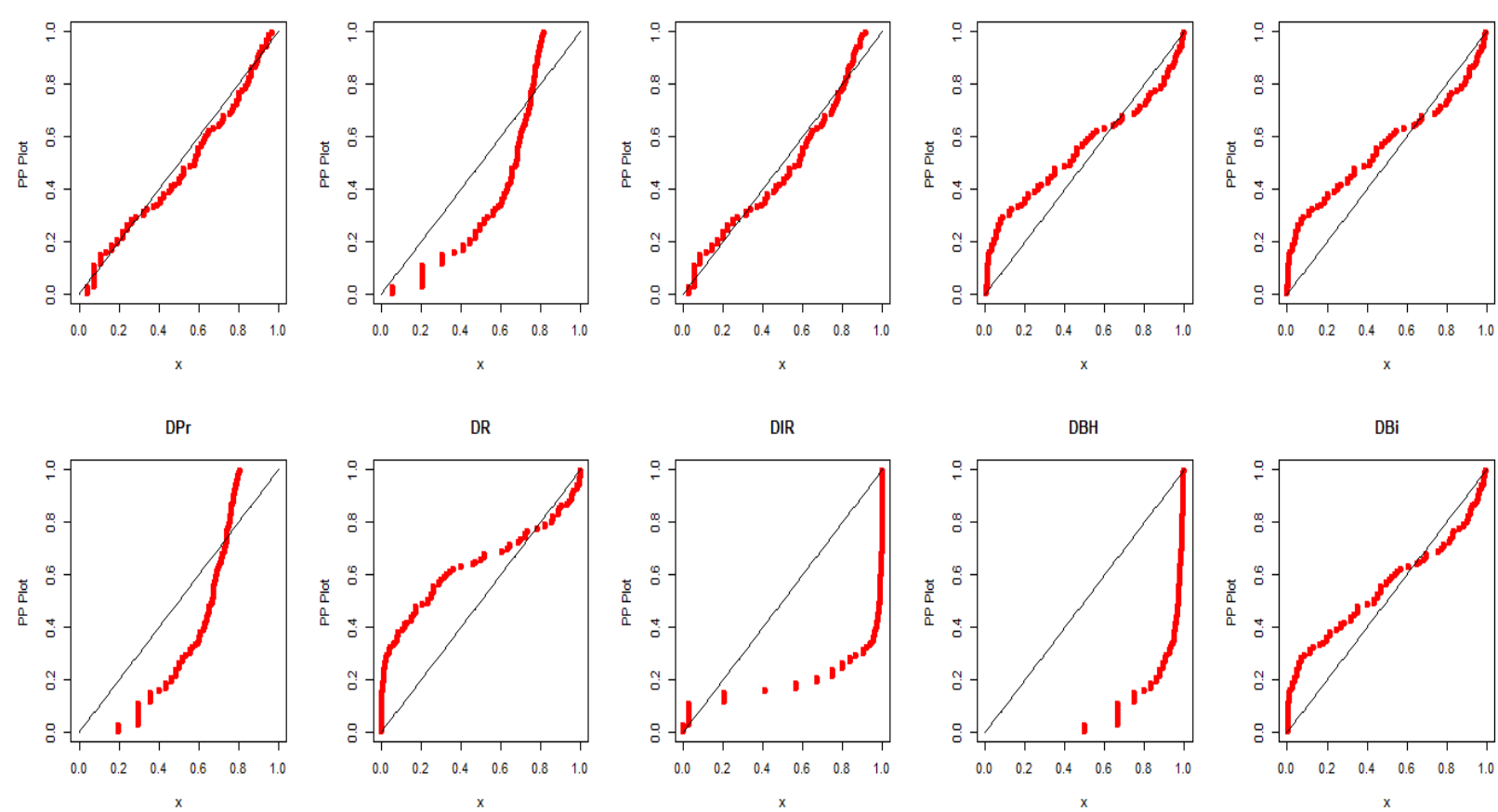

Figure 3: The PP plots of the competing discrete distributions for the number of deaths in Pakistan 


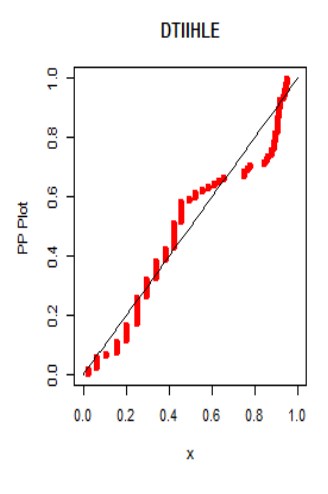

$\mathrm{DPr}$

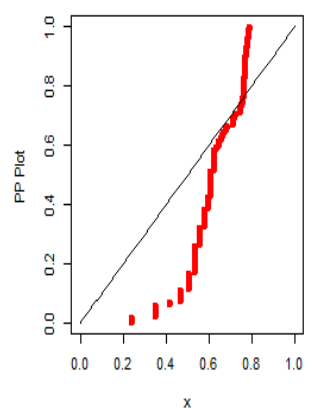

DBXII

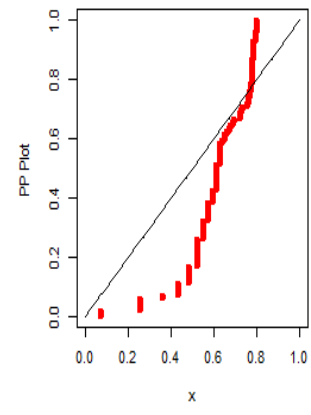

DR

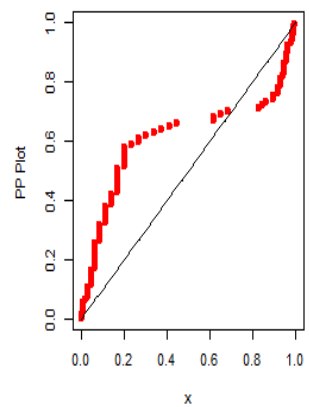

DLoL

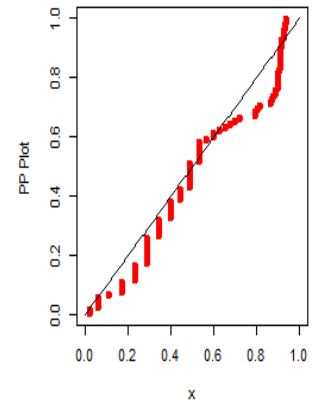

DIR

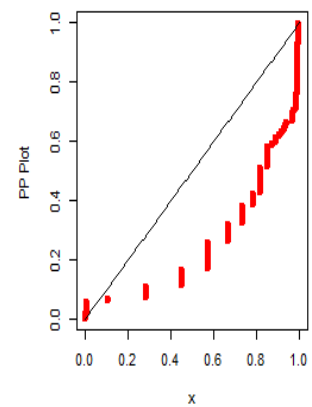

DL

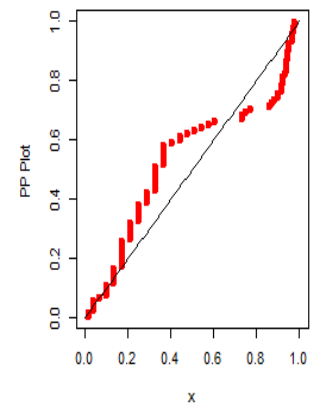

DBH

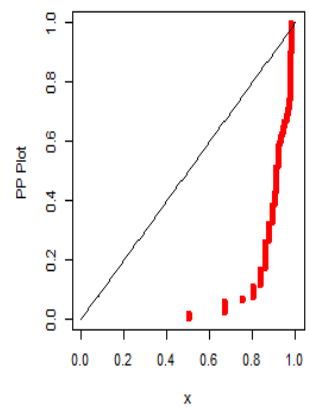

NDL

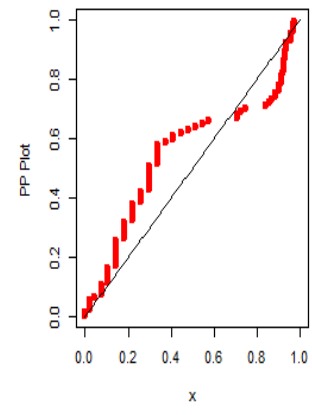

$\mathrm{DBi}$

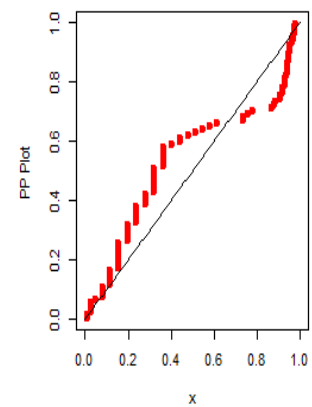

Figure 4: The PP plots of the competing discrete distributions for the number of deaths in Saudi Arabia

\section{Conclusion}

In this article, a two-parameter discrete distribution is proposed to model COVID-19 new cases in Pakistan and Saudi Arabia, called the discrete Type-II half-logistics exponential (DTIIHLE) distribution. Several mathematical properties of the DTIIHLE model are discussed. Its parameters have been estimated by using the maximum likelihood approach. A simulation study was carried out to check the performance of parameters based on MSEs and CP. The DTIIHLE model is utilized to model two real-life data sets about the number of COVID-19 deaths in Pakistan and Saudi Arabia due to COVID-19. The newly DTIIHLE model is important to elaborate on the existing discrete distributions in the literature. It has the lowest goodness-of-fit measures values among all discrete competing models. Hence, the proposed model is best among competitive distributions.

\section{References}

1. Alamatsaz, M. H., Dey, S., Dey, T. and Harandi, S. S. (2016). Discrete generalized Rayleigh distribution. Pakistan journal of statistics, 32, 1-20.

2. Al-Babtain, A. A., Ahmed, A. H. N. and Afify, A. Z. (2020). A new discrete analog of the continuous lindley distribution, with reliability applications. Entropy, 22, p.603.

3. AL-Huniti, A. A. and AL-Dayian, G. R. (2012). Discrete Burr type 3 distribution. American Journal of Mathematics and Statistics, 2, 145-152.

4. Aljohani, H. M., Akdogan, Y., Cordeiro, G. M. and Afify, A. Z. (2021). The uniform Poisson-Ailamujia distribution: actuarial measures and applications in biological science. Symmetry, 13, 1258.

5. Almazah, M. M. A., Erbayram, T., Akdogan, Y., AL Sobhi, M. M. and Afify, A. Z. (2021). A new extended geometric distribution: properties, regression model, and actuarial applications. Mathematics, $9,1336$.

6. Altun, E., El-Morshedy, M. and Eliwa, M. S. (2020). A study on discrete Bilal distribution with properties and applications on integer-valued autoregressive process. Revstat. Stat. J, 18, 70-99. 
7. Bakouch, H. S., Jazi, M. A. and Nadarajah, S. (2014). A new discrete distribution. Statistics, 48, 200240.

8. Chakraborty, S. (2015). Generating discrete analogues of continuous probability distributions-a survey of methods and constructions. Journal of Statistical Distributions and Applications, 2, 1-30.

9. Chakraborty, S. and Chakravarty, D. (2012). Discrete gamma distribution: properties and Parameter Estimation. Commun. Stat. Theory and Methods, 41, 3301-3324.

10. Eldeeb, A. S., Ahsan-ul-Haq, M. and Babar, A. (2021). A discrete analog of inverted Topp-Leone distribution: properties, estimation and applications. International Journal of Analysis and Applications, 19, 695-708.

11. Elgarhy, M., Ahsan-ul-Haq, M. and Perveen, I. (2019). Type II half logistic exponential distribution with applications. Annals of Data Science, 6, 245-257.

12. El-Morshedy, M., Eliwa, M. S. and Altun, E. (2020). Discrete Burr-Hatke distribution with properties, estimation methods and regression model. IEEE Access, 8, 74359-74370.

13. Ghosh, T., Roy, D. and Chandra, N. K. (2013). Reliability approximation through the discretization of random variables using reversed hazard rate function. International Journal of Mathematical, Computational, Statistical, Natural and Physical Engineering, 796-100.

14. Good, I. J. (1953) The population frequencies of species and the estimation of population parameters. Biometrika, 40, 237-264.

15. Hussain, T. and Ahmad, M. (2014). Discrete inverse Rayleigh distribution. Pakistan Journal of Statistics, 30, 203-222.

16. Inusah, S. and Kozubowski, T. J. (2006). A discrete analogue of the Laplace distribution. Journal of Statistical Planning and Inference, 136, 1090-1102.

17. Jazi, M. A., Lai, C. D. and Alamatsaz, M.H. (2010). A discrete inverse Weibull distribution and estimation of its parameters. Statistical Methodology, 7, 121-132.

18. Kemp, A. W. (1997) Characterizations of a discrete normal distribution. Journal of Statistical Planning and Inference, 63, 223-229.

19. Kozubowski, T. J. and Inusah, S. (2006). A skew Laplace distribution on integers. The Institute of Statistical Mathematics. ASIM, 58, 555-571.

20. Krishna, H. and Pundir, P. S. (2007). Discrete Maxwell distribution. InterStat, 3, 1-15.

21. Krishna, H. and Pundir, P. S. (2009). Discrete Burr and discrete Pareto distributions. Statistical Methodology, 6, 177-188.

22. Kulasekera, K. B. and Tonkyn, D. W. (1992) A new discrete distribution, with applications to survival, dispersal and dispersion. Commun. Stat. Simulation and Computation, 21, 499-518.

23. Li, Q., Guan, X., Wu, P., Wang, X., Zhou, L., Tong, Y., Ren, R., Leung, K. S., Lau, E. H., Wong, J. Y. and Xing, X. (2020). Early transmission dynamics in Wuhan, China, of novel coronavirus infected pneumonia. New England journal of medicine, 382, 1199-1207.

24. Nakagawa, T. and Osaki, S. (1975). The discrete Weibull Distribution. IEEE Transactions on Reliability, 24, 300-301.

25. Roy, D. (2004). Discrete Rayleigh distribution. IEEE Trans. Reliab., 53, 255-260.

26. Sato, H., Ikota, M., Sugimoto, A. and Masuda, H. (1999) A new defect distribution metrology with a consistent discrete exponential formula and its applications. IEEE Trans Semicond Manuf., 12, 409418. 